\title{
FRATURA TORACOLOMBAR TIPO EXPLOSÃO: CLASSIFICAÇÃO DE MCCORMACK E FALHA DE INSTRUMENTAÇÃO POSTERIOR
}

\author{
THORACOLUMBAR BURST FRACTURE: \\ LOAD SHARING CLASSIFICATION AND POSTERIOR INSTRUMENTATION FAILURE
}

Osmar Avanzi', Elcio Landim², Robert Meves ${ }^{3}$, Maria Fernanda Caffaro 4 , Felipe de Albuquerque Araujo Luyten ${ }^{5}$, Antonio Alexandre Faria ${ }^{5}$

\section{RESUMO}

Objetivos: Verificar a associação entre o colapso sagital, falha da instrumentação posterior e a classificação de McCormack nos portadores de fratura toracolombar tipo explosão submetidos à artrodese posterior maior do que um nível acima e um abaixo da fratura. Métodos: Estudo retrospectivo com base em prontuários e radiografias de 22 pacientes operados entre janeiro de 1990 e dezembro de 2006. Os parâmetros avaliados foram: piora da cifose (Cobb), disfunção neurológica (Frankel) e falha do tratamento cirúrgico (quebra ou soltura do implante). Resultados: Não houve correlação entre o colapso sagital e a pontuação da classificação de McCormack ( $r=0,221 ; P=0,322)$. Verificamos ainda inexistência de associação entre a falha do implante posterior e a pontuação desta classificação $(p=0,85)$. Conclusão: Nossos achados sugerem a falta de aplicabilidade desta classificação em pacientes submetidos a artrodese posterior maior do que um nível acima e um abaixo da fratura.

Descritores - Fraturas da coluna vertebral; Vértebras torácicas; Vértebras lombares; Resultado de tratamento; Humanos; Suporte de carga

\section{ABSTRACT}

Objectives: To assess the relationship between sagittal collapse, implant failure and load sharing classification of patients suffering a thoracolumbar burst fracture treated with posterior instrumentation. Methods: Retrospective review of radiographs and medical records of 26 patients operated on between January 1990 and December 2006. Kyphotic worsening (Cobb), neurologic dysfunction (Frankel) and the failure of surgical treatment (implant breaking or loosening) were studied. Results: No relationship was found between kyphotic deformity and higher scores in the load sharing classification ( $r=0,221 ; P=0,322)$. No relationship was found between implant failure and this classification. $(p=0.85)$. Conclusion: Our findings suggest that this classification is not applicable in patients with an arthrodesis larger than one unit above and below the fracture.

Keywords - Spinal fractures; Thoracic vertebrae; Lumbar vertebrae; Treatment outcome; Human; Weight-Bearing

\footnotetext{
1 - Professor Doutor Adjunto do Departamento de Ortopedia e Traumatologia da Faculdade de Ciências Médicas da Santa Casa de São Paulo.

2 - Consultor Sênior do Grupo de Coluna do Departamento de Ortopedia e Traumatologia da Faculdade de Ciências Médicas da Santa Casa de São Paulo.

3 - Professor Doutor Assistente do Departamento de Ortopedia e Traumatologia da Faculdade de Ciências Médicas da Santa Casa de São Paulo.

4 - Professora Doutora e Instrutora do Departamento de Ortopedia e Traumatologia da Faculdade de Ciências Médicas da Santa Casa de São Paulo.

5 - Médicos Residentes do Departamento de Ortopedia e Traumatologia da Faculdade de Ciências Médicas da Santa Casa de São Paulo.
}

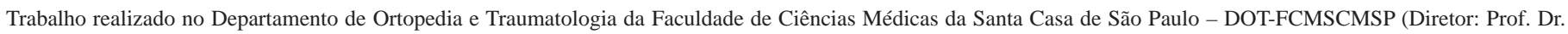
Osmar Avanzi).

Correspondência: Robert Neves - Rua Dr. Cesário Mota Júnior, 112 - 01277-900 - São Paulo - SP - Brasil - E-mail: robertmeves@hotmail.com 


\section{INTRODUÇÃO}

A fratura toracolombar é a lesão esquelética mais frequente do esqueleto axial - responsável por cerca de $90 \%$ de todas as fraturas na coluna ${ }^{(1,2)}$. Em função da rigidez das articulações costotransversárias da coluna torácica, o fulcro da movimentação no momento do trauma acontece no segmento toracolombar da coluna, onde ocorre a fratura na grande maioria dos casos. A presença de sintomas neurológicos pode atingir até $40 \%$ dos pacientes, justificando a alta morbidade destas lesões ${ }^{(1-3)}$. Em torno de $50 \%$ dos pacientes, estas lesões são decorrentes de quedas de altura, $45 \%$ por acidentes automobilísticos e $5 \%$ causadas por trauma direto ${ }^{(4)}$.

Várias classificações na literatura descrevem os tipos de fraturas da transição toracolombar ${ }^{(5-18)}$. Holdsworth $^{(6)}$ e Denis ${ }^{(11)}$ publicaram a classificação das fraturas toracolombares com base em colunas. A fratura tipo explosão apresenta, caracteristicamente, lesão esquelética da coluna anterior e média que, do ponto de vista ósseo, corresponde ao corpo vertebral. O aspecto na tomografia axial (TAC) típico é o fragmento do corpo migrado para o interior do canal vertebral ${ }^{(12)}$.

McCormack et al ${ }^{(17)}$ desenvolveram a classificação conhecida como Load Sharing Classification, que pontua a gravidade da lesão esquelética da fratura do corpo vertebral conforme a distância entre os fragmentos do corpo vertebral, o grau de correção da cifose após a cirurgia por via posterior e o colapso do corpo vertebral no plano sagital. Para esses autores, quanto maior a pontuação da fratura maior o risco de falha do implante posterior após artrodeses curtas - um nível acima e um nível abaixo da fratura - com uso de parafusos pediculares. Por isso, indicam também artrodese anterior nos pacientes com pontuação maior ou igual a sete.

Na prática, muitos cirurgiões advogam intuitivamente a realização de artrodese anterior após a artrodese posterior quando existe grande lesão esquelética do corpo vertebral, mesmo após instrumentações mais longas. Faltam estudos, no entanto, que analisem a relação entre a classificação de McCormack et $a l^{(17)}$ e o colapso sagital nos pacientes submetidos a tratamento cirúrgico com maiores níveis de artrodese (maior do que um acima e um abaixo da vértebra fraturada) ${ }^{(4,19)}$.

Diante disso, realizamos estudo retrospectivo para verificar a aplicabilidade da classificação de McCormack et $a l^{(17)}$ após a artrodese posterior longa nesses pacientes.

\section{MÉTODOS}

Após aprovação do Comitê de Ética em Pesquisa em Seres Humanos da nossa Instituição foi realizado estudo retrospectivo com base em dados de prontuários, radiografias e de TAC dos portadores de fratura toracolombar tipo explosão, segundo Denis ${ }^{(11,12)}$, recentes (< 10 dias) operados de janeiro de 1990 a dezembro de 2006 mediante instrumentação metálica e artrodese posterior. Incluímos pacientes com fratura tipo explosão localizadas na região toracolombar envolvendo apenas um nível e com seguimento maior do que seis meses. Excluímos fraturas por arma de fogo, patológicas ou tratadas conservadoramente. A gravidade da lesão esquelética foi pontuada conforme McCormack et al ${ }^{(17)}$ (Tabela 1).

Tabela 1 - Classificação de McCormack (load sharing)

\begin{tabular}{c|c|c|c}
\hline Pontuação & 1 ponto & 2 pontos & 3 pontos \\
\hline Colapso sagital & $30 \%$ & $>30 \%$ & $60 \%$ \\
\hline Deslocamento & $1 \mathrm{~mm}$ & $2 \mathrm{~mm}$ & $>2 \mathrm{~mm}$ \\
\hline Correção & 3 graus & 9 graus & 10 graus \\
\hline Total & 3 pontos & 6 pontos & 9 pontos \\
\hline Fonte: McCormack T. Karaikovic E. Gaines RW.The load sharing classification of spine fractures.
\end{tabular}

Fonte: McCormack T, Karaikovic E, Gaines RW. The load sharing classification of spine fractures. Spine 1994;19(15):1741-4.

A mensuração radiográfica da cifose no período pré e pós-operatório foi realizada pelo método de Ulmar ${ }^{(20)}$ e o estreitamento do canal vertebral por meio do diâmetro sagital médio ${ }^{(13)}$. A gravidade da disfunção neurológica foi categorizada pela escala de Frankel $^{(21)} \mathrm{em}$ tipo A (ausência de força muscular e sensibilidade), B (sensibilidade preservada), C (sensibilidade preservada com força muscular não funcional para o paciente), D (sensibilidade preservada com força muscular funcional) e tipo E (exame neurológico normal).

A análise estatística foi realizada com base no programa SPSS (Statistical Package for Social Sciences), versão 13.0. Foi adotado nível de significância de 5\% $(0,050)$ para aplicação dos testes estatísticos.

\section{RESULTADOS}

Preencheram os critérios de inclusão deste estudo 22 pacientes - 13 do sexo masculino e nove do feminino. Sete pacientes apresentaram déficit neurológico inicial: dois com Frankel C, três com D, um com B e um com A.

O nível de fratura mais acometido foi L1 em 12 pacientes, seguido por L2 em quatro, L3 em três, T12 em dois e L4 em um. A cifose pós-traumática variou de $3^{\circ}$ a $37^{\circ}$ (média de $17^{\circ} \pm 8,2$ ) no período pré-operatório. 
A porcentagem de acometimento do canal variou de 9 a $70 \%$ (média de $42,2 \% \pm 14,5$ ). Doze pacientes foram submetidos à instrumentação híbrida com ganchos e parafusos, três pacientes com parafusos pediculares e sete com instrumentação segmentar (Hartchill ou HarriLuque). Houve falha da instrumentação em dois pacientes submetidos a dois níveis de artrodese acima e um abaixo da fratura (Quadro 1).

Quadro 1 - Distribuição dos pacientes conforme os níveis de artrodese acima e abaixo da fratura e falha da instrumentação posterior

\begin{tabular}{|c|c|c|}
\hline Tipo artrodese & Número de pacientes & Falha do implante \\
\hline 2 e 1 & 8 & 2 \\
\hline 2 e 2 & 6 & 0 \\
\hline 3 e 2 & 8 & 0 \\
\hline Total & 22 & 2 \\
\hline
\end{tabular}

Em todos os pacientes houve consolidação radiográfica da artrodese no final do seguimento, mesmo nos com falha do implante (Figuras 1 a 3). Quanto ao quadro neurológico houve melhora em pelo menos um grau da escala de Frankel em cinco pacientes (Quadro 2).

Quadro 2 - Distribuição dos pacientes conforme Frankel

\begin{tabular}{|c|c|c|c|c|c|}
\hline Frankel & A & B & C & D & E \\
\hline A & & 1 & & & \\
\hline B & & & 1 & & \\
\hline C & & & & 2 & \\
\hline D & & & & 2 & 1 \\
\hline E & & & & & 15 \\
\hline
\end{tabular}

Fonte: S.A.M.E.

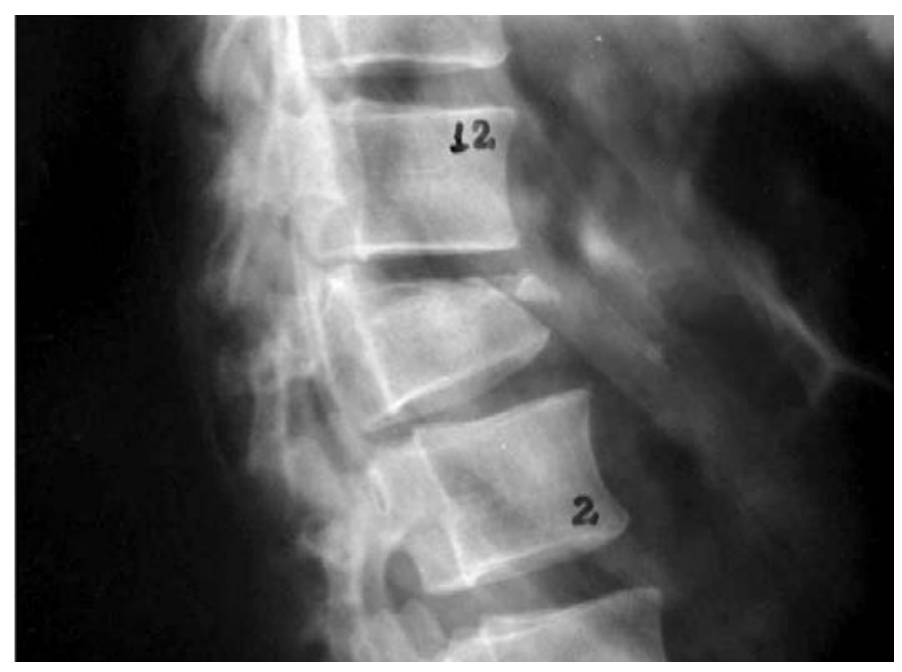

Fonte: S.A.M.E.

Figura 1 - Radiografia inicial em perfil de paciente portadora de fratura tipo explosão L1 neurologicamente intacta

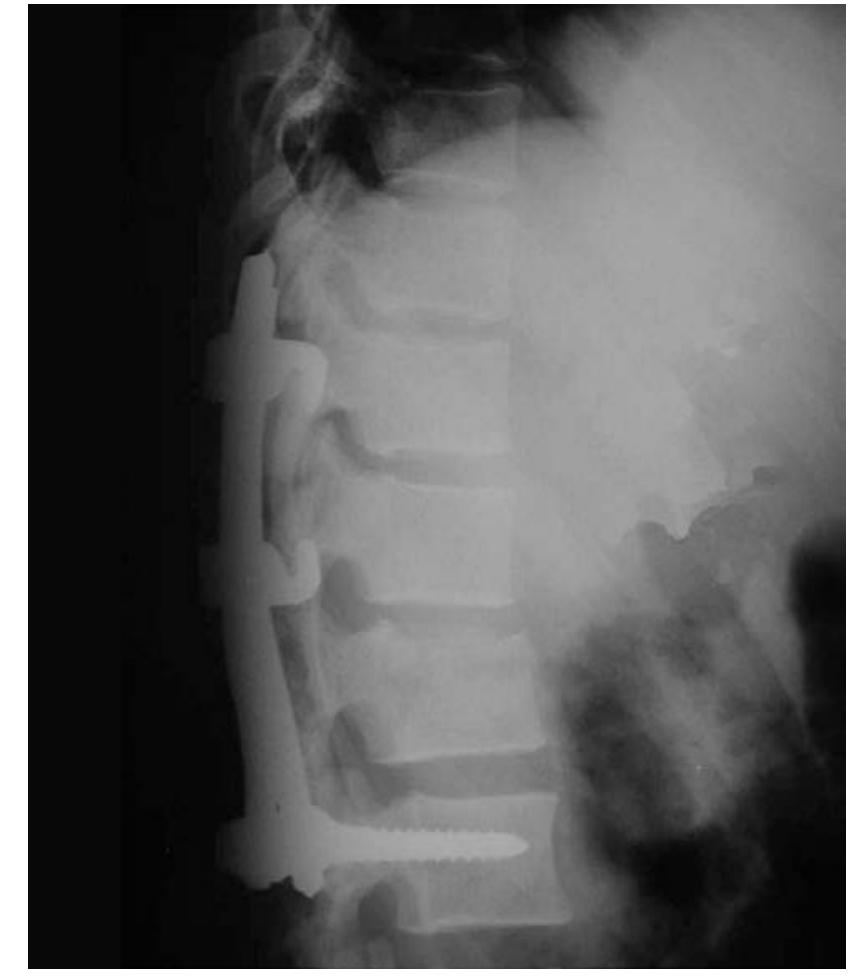

Fonte: S.A.M.E.

Figura 2 - Radiografia pós-operatória imediata

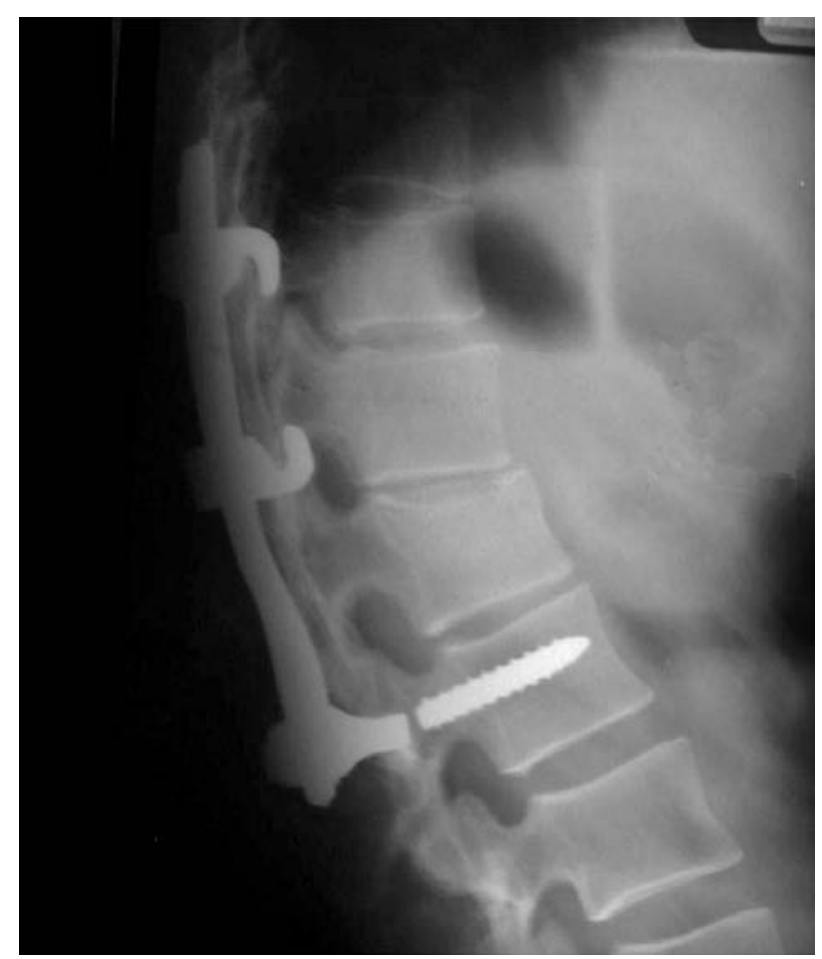

Fonte: S.A.M.E.

Figura 3 - Observar a falha do implante, causando dor na proeminência do implante

Constatamos que não houve associação entre a falha de instrumentação posterior e a média da pontuação da classificação de McCormack (Tabela 2). 
Tabela 2 - Associação entre colapso sagital e classificação de McCormack

\begin{tabular}{c|c|c|c|c|c}
\hline & $\begin{array}{c}\text { Falha do } \\
\text { implante }\end{array}$ & $\mathbf{n}$ & Média & $\begin{array}{c}\text { Desvio } \\
\text { padrão }\end{array}$ & $\begin{array}{c}\text { Significância } \\
(\mathbf{p})\end{array}$ \\
\hline \multirow{2}{*}{ Pontuação } & $\operatorname{sim}$ & 2 & 4,5 & 0,71 & \\
\cline { 2 - 5 } & não & 20 & 6,7 & 1,63 & 0,85 \\
\hline
\end{tabular}

Fonte: S.A.M.E. Teste de Mann-Withney.

Observamos ainda que não houve correlação entre o colapso sagital e a pontuação da classificação McCormack (Figura 4).

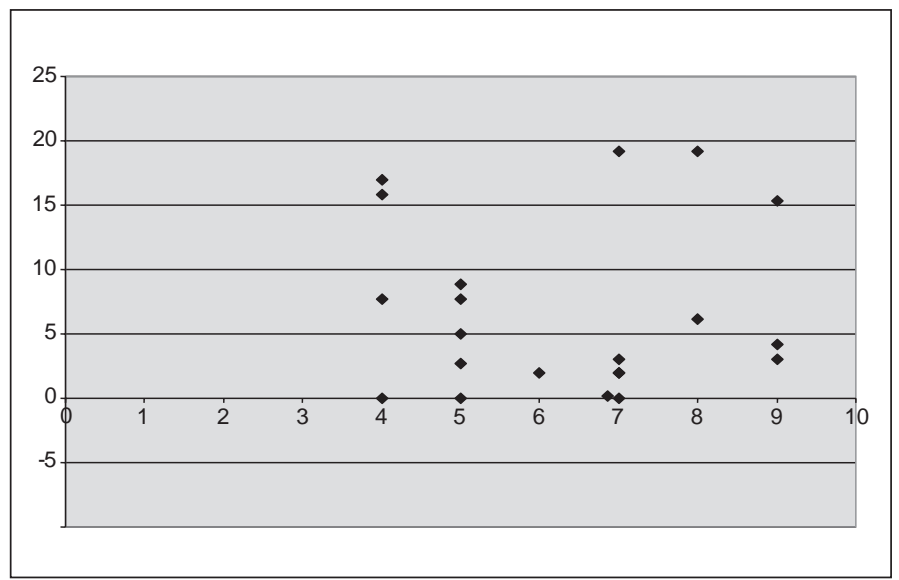

Fonte: S.A.M.E. Correlação de Spearman. $R=0,221 ; P=0,322$.

Figura 4 - Correlação entre a pontuação da classificação McCormack e colapso sagital

\section{DISCUSSÃO}

A fratura toracolombar tipo explosão é frequentemente observada nos pacientes vítimas de acidente de trânsito ou queda de altura. Nos países em desenvolvimento, a queda de altura responde ainda pela grande maioria dos casos $^{(4,11,22-24)}$. Dados demográficos reportados em vários trabalhos na literatura apontam o típico portador desta lesão: jovem do sexo masculino e com fratura na coluna toracolombar ${ }^{(5,13,25-27)}$. A cifose pós-traumática sintomática com ou sem disfunção neurológica representa a significativa morbidade para o paciente e a repercussão socioeconômica para a sociedade ${ }^{(1,10)}$.

Várias classificações são descritas na literatura com o intuito de categorizar mais detalhadamente estas fraturas, definir instabilidade ou auxiliar na escolha da melhor opção de tratamento ${ }^{(13-16)}$. Holdsworth ${ }^{(6,7)} \mathrm{e}$ Denis $^{(12)}$, contudo, explicaram a característica básica desta fratura: a cominuição do corpo vertebral com frag- mento ósseo migrado para o interior do canal vertebral. Esta imagem, observada na TAC, é o aspecto clássico considerado para análise dos desfechos clínicos e radiográficos na grande maioria das publicações ${ }^{(11,12,28)}$.

A Classificação de McCormack et $a l^{(17)}$ pontua a gravidade da lesão esquelética, segundo a gravidade do encunhamento e da cominuição do corpo vertebral, com o intuito de predizer a falha de instrumentação após a artrodese curta posterior incluindo um nível vertebral acima e um abaixo da vértebra fraturada com o uso de parafusos pediculares. Sasso et $a l^{(19)}$ e Parker et $a l^{(18)}$, descreveram a validade do método para a indicação da artrodese anterior associada em casos com pontuação maior ou igual a sete a fim de evitar a falha de instrumentação curta posterior. Aligizakis et al $^{(1)}$ extrapolaram a aplicabilidade do método para prever o colapso sagital e a função dos pacientes após o tratamento conservador nestas fraturas.

A literatura mostra que frequentemente a artrodese anterior é associada a posterior quando há grande cominuição do corpo vertebral ${ }^{(4,11,12,25)}$, independente dos níveis escolhidos acima e abaixo da vértebra fraturada. Apesar disso, falta análise da aplicabilidade desta classificação neste grupo de pacientes.

Os nossos achados, observados em pacientes tratados com artrodeses com mais de um nível acima e pelo menos um nível abaixo da vértebra lesionada, sugeriram inexistência de correlação entre o colapso sagital (piora da cifose) e a gravidade da fratura conforme os critérios de McCormack et $a^{(17)}$. Estes achados sugerem a falta de aplicabilidade da classificação de McCormack et $a l^{(17)}$ nos pacientes operados mediante artrodese posterior com artrodeses mais longas. A falha de instrumentação foi quebra em um paciente (Figuras 1 a 3) e soltura em outro. Não se constatou falha da instrumentação nas estratégias com dois níveis acima e dois níveis abaixo da fratura e de três níveis acima e dois níveis abaixo da fratura (Quadro 2).

Algumas limitações desta pesquisa devem ser lembradas. Tratou-se de estudo retrospectivo com inerente dificuldade de controle de variáveis, como a estratégia de instrumentação posterior. Os vários tipos de instrumentação - instrumentação segmentar, terceira geração híbrida e com uso de parafusos pediculares - e níveis de artrodese posterior diminuem o número de nossa amostra para a validação e conclusão estatística definitiva dos resultados. Por isso, não foi avaliada a diferença estatística dos desfechos entre as diferentes técnicas e níveis 
de artrodese das instrumentações segmentares utilizadas nesta série de casos. Ademais, o desfecho utilizado foi radiográfico, o que não significa que a falha represente obrigatoriamente piores resultados funcionais. Apesar disso, nosso estudo é o primeiro a enfatizar que estratégias de instrumentação com artrodese maiores do que um nível acima e um nível abaixo da fratura devem ser analisadas de forma diferente no que tange à classificação de McCormack.

Estudos randomizados controlados com série maior de casos podem auxiliar a definir a melhor estratégia de instrumentação nestes pacientes, com a finalidade de evitar a falha da instrumentação. A divisão em grupos conforme a pontuação da classificação de McCormack et al $^{(17)}$ pode, ainda, definir grupos mais homogêneos para análise dos desfechos clínicos e radiográficos em estudos futuros nestes pacientes.

\section{CONCLUSÃO}

Nossos achados sugerem a falta de relação entre a falha de instrumentação, colapso sagital e a pontuação da classificação de McCormack nos pacientes portadores de fratura toracolombar tipo explosão submetidos à artrodese maiores do que um nível acima e um abaixo da fratura.

\section{REFERÊNCIAS}

1. Aligizakis A, Katonis P, Stergiopoulos K, Galanakis I, Karabekios S, Hadjipavlou A. Functional outcome of burst fractures of the thoracolumbar spine managed non-operatively, with early ambulation, evaluated using the load sharing classification. Acta Orthop Belg. 2002;68(3):279-87.

2. Haher TR, Felmy W, Baruch H, Devlin V, Welin D, O'Brien M, et al. The contribution of the three columns of the spine to rotational stability. A biomechanical model. Spine (Phila Pa 1976). 1989;14(7):663-9.

3. Aebi M, Etter C, Kehl T, Thalgott J. Stabilization of the lower thoracic and lumbar spine with the internal spinal skeletal fixation system. Indications, techniques, and first results of treatment. Spine (Phila Pa 1976). 1987;12(6):544-51.

4. Defino HL, Rodriguez-Fuentes AE. Treatment of fractures of the thoracolumbar spine by combined anteroposterior fixation using the Harms method. Eur Spine J. 1998;7(3):187-94.

5. Watson-Jones R. Fractures and joint injuries. 3rd ed. Baltimore, Williams \& Wilkins; 1943.

6. Holdsworth FW. Fractures, dislocations, and fracture-dislocations of the spine. J Bone Joint Surg Br. 1963;45(1):6-20.

7. Holdsworth F. Fractures, dislocations, and fracture-dislocations of the spine. $\mathrm{J}$ Bone Joint Surg Am. 1970;52(8):1534-51.

8. Kelly RP, Whitesides TE Jr. Treatment of lumbodorsal fracture-dislocations. Ann Surg. 1968;167(5):705-17.

9. Bedbrook GM. Treatment of thoracolumbar dislocation and fractures with paraplegia. Clin Orthop Relat Res. 1975;(112):27-43.

10. Lucas JT, Ducker TB. Motor classification of spinal cord injuries with mobility, morbidity and recovery indices. Am Surg. 1979;45(3):151-8.

11. Denis $F$. The three column spine and its significance in the classification of acute thoracolumbar spinal injuries. Spine (Phila Pa 1976). 1983;8(8):817-31.

12. Denis F. Spinal instability as defined by the three-column spine concept in acute spinal trauma. Clin Orthop Relat Res. 1984;(189):65-76.

13. McAfee PC, Yuan HA, Lasda NA. The unstable burst fracture. Spine (Phila Pa 1976). 1982;7(4):365-73.

14. Ferguson RL, Allen BL Jr. A mechanistic classification of thoracolumbar spine fractures. Clin Orthop Relat Res. 1984;(189):77-88.

15. Gertzbein SD. Spine update. Classification of thoracic and lumbar fractures. Spine (Phila Pa 1976). 1994;19(5):626-8.
16. Magerl F, Aebi M, Gertzbein SD, Harms J, Nazarian S. A comprehensive classification of thoracic and lumbar injuries. Eur Spine J. 1994;3(4):184-201.

17. McCormack T, Karaikovic E, Gaines RW. The load sharing classification of spine fractures. Spine (Phila Pa 1976). 1994;19(15):1741-4.

18. Parker JW, Lane JR, Karaikovic EE, Gaines RW. Successful short-segment instrumentation and fusion for thoracolumbar spine fractures: a consecutive 41/2-year series. Spine (Phila Pa 1976). 2000;25(9):1157-70.

19. Sasso RC, Renkens K, Hanson D, Reilly T, McGuire RA Jr, Best NM. Unstable thoracolumbar burst fractures: anterior-only versus short-segment posterior fixation. J Spinal Disord Tech. 2006;19(4):242-8.

20. Ulmar B, Gühring M, Schmälzle T, Weise K, Badke A, Brunner A. Inter- and intra-observer reliability of the Cobb angle in the measurement of vertebral, local and segmental kyphosis of traumatic lumbar spine fractures in the lateral X-ray. Arch Orthop Trauma Surg. 2010 Apr 28. [Epub ahead of print].

21. Frankel HL, Hancock DO, Hyslop G, Melzak J, Michaelis LS, Ungar GH, et al. The value of postural reduction in the initial management of closed injuries of the spine with paraplegia and tetraplegia. I. Paraplegia. 1969;7(3):179-92.

22. Wang XY, Dai LY, Xu HZ, Chi YL. The load-sharing classification of thoracolumbar fractures: an in vitro biomechanical validation. Spine (Phila Pa 1976). 2007;32(11):1214-9.

23. Scholl BM, Theiss SM, Kirkpatrick JS. Short segment fixation of thoracolumbar burst fractures. Orthopedics. 2006;29(8):703-8.

24. Avanzi O, Chih LY, Meves R. Avaliação do tratamento cirúrgico da fratura toracolombar com material de terceira geração. Rev Bras Ortop. 2002;37(6):226-32.

25. Avanzi O, Chih LY, Meves R, Silber MF. Avaliação do tratamento cirúrgico tardio na fratura toracolombar tipo tipo explosão. Coluna. 2002;1(2):88-93.

26. Meves R, Avanzi O. Correlation between neurological deficit and spinal canal compromise in 198 patients with thoracolumbar and lumbar fractures. Spine (Phila Pa 1976). 2005;30(7):787-91.

27. Meves R, Avanzi O. Correlation among canal compromise, neurologic deficit, and injury severity in thoracolumbar burst fractures. Spine (Phila Pa 1976). 2006;31(18):2137-41.

28. Avanzi O, Chih LY, Meves R, Silber MF. Avaliação tomográfica do fragmento retropulsado nas fraturas toracolombares tipo explosão. Coluna. 2006;5(4):247-52. 